\title{
Android Application to Read and Record Data from Multiple Sensors
}

\author{
Vishesh S ${ }^{1}$, Manu Srinath ${ }^{1}$, Ranjitha Ravishankar ${ }^{2}$, Ranjan Ravishankar ${ }^{3}$, Ashrith Pradeep ${ }^{4}$, Jeevan HS $^{\mathbf{5}}$ \\ B.E, Department of Telecommunication Engineering, BNM Institute of Technology, Bangalore, India ${ }^{1}$ \\ Student, Department of Telecommunication Engineering, BNM Institute of Technology, Bangalore, India ${ }^{2}$ \\ Student, Department of Computer Science and Engineering, Atria Institute of Technology, Bangalore, India ${ }^{3}$
}

Student, Department of Computer Science and Engineering, Visvesvaraya Technological University, Bangalore, India ${ }^{4}$

Student, Department of Information Science and Engineering, BNM Institute of Technology, Bangalore, India ${ }^{5}$

\begin{abstract}
The increasing capabilities of smart phones have led to the replacement of PC's in almost all real-time scenarios. A Smartphone is a mobile/portable personal computer with a swift processor, advanced mobile operating system, huge memory space and a platform for many real-time applications-business, accounting, entertainment, scientific, recreation, education, finance, social networking, news and many other applications suited to the 21 st century impatient world. In the early inception of mobile technology development, mobile phones were elite devices primarily used by middle and upper class people. But with more and more Smartphones manufacturers entering the market, the price of a Smartphone is so affordable [1] that today about half the adult population owns a Smartphone. [2] Android phones are increasingly becoming cheaper [3], better and more intelligent. Android is a mobile OS developed by Google, based on the Linux kernel. [4] In this paper, we develop an android application which can monitor the realtime values of any electronic/optical/acoustic sensor on android powered Smartphone. The mobile application is well suited to the scientific community, engineers and professionals/amateurs in their real-time monitoring of sensed value. The stream of real time data is sent wirelessly to the Smartphone via Bluetooth or Wi-Fi. In this paper, we also present to you the ADLC model which includes feasibility study, Requirement analysis, Design and implementation, integration and testing, operation and maintenance and marketing.
\end{abstract}

Keywords: Smartphone, operating system, Linux kernel, ADLC model, Bluetooth, Wi-Fi.

\section{INTRODUCTION}

Mobile communication is so integrated into our lives that many people feel uncomfortable without a cell phone. Earlier, when a mobile phone was launched into the market, it had basic functionalities like calling and SMS services. But today is the era of Smartphones. A Smartphone is a mobile personal computer with an advanced mobile operating system. The Smartphones of the present day have a swift processor, RAM of few gigabytes, ROM of 100s of gigabytes, multi-core processor architecture, USB port and other I/O ports. A Smartphone can easily be connected to the internet via mobile network data modem or Wi-Fi. One of the main characteristics of a Smartphone is their screen. It usually fills most of the phone's front surface. The versatility of Smartphone operating systems like Android, IOS [5] etc... makes the Smartphones of the $21^{\text {st }}$ century to havea broad-minded approach and nurtures the mélange needs of the diversified $21^{\text {st }}$ century world market place. These operating systems not only provide basic telephony and data applications but infinite real time mobile applications which can be developed and released into the market by the OS creator himself or by millions of third party mobile application coders. Meaning an android or IOS application can be developed by any individual and proudly released into the market, with proper knowledge of coding. This opens up infinite possibilities as each programmer or application builder, is himself an institute of discoveries and updates. In this paper, we have developed an android application which can monitor the real time values of any electronic/ optical/ acoustic sensor. We have followed the Software Development Life Cycle (SDLC) or Application Development Life Cycle (ADLC) model. Figure 1shows the steps involved in a SDLC model.

They are:

- Requirements analysis

- Feasibility study

- Design and implementation

- Integration and testing

- Operation and maintenance

- Marketing 


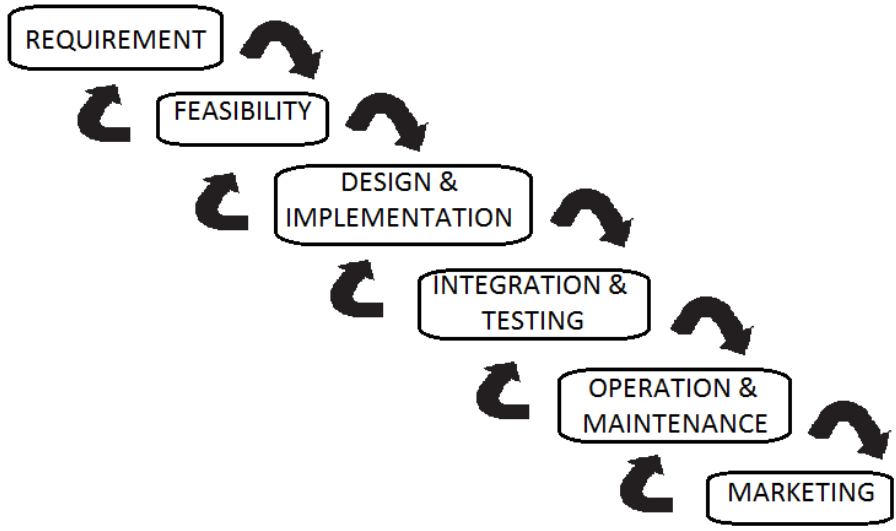

Figure 1Software Development Lifecycle or Application Development Lifecycle

In this model, anywhere (at any block) and anytime, backward traversing can be achieved. This is done in case of an error in the previous blocks or a demand for an update of the existing application. SDLC modelling is a flexible and multifaceted approach in application development. A stream of sensor data/values is wirelessly transmitted using Bluetooth or Wi-Fi to the android Smartphone after processing by a microcontroller. Figure 2 shows the block diagram of wireless monitoring of sensor values using Smartphone with an android application installed in it, which is capable to carry out the intended application of sensor value(analog or digital) monitoring and recording.

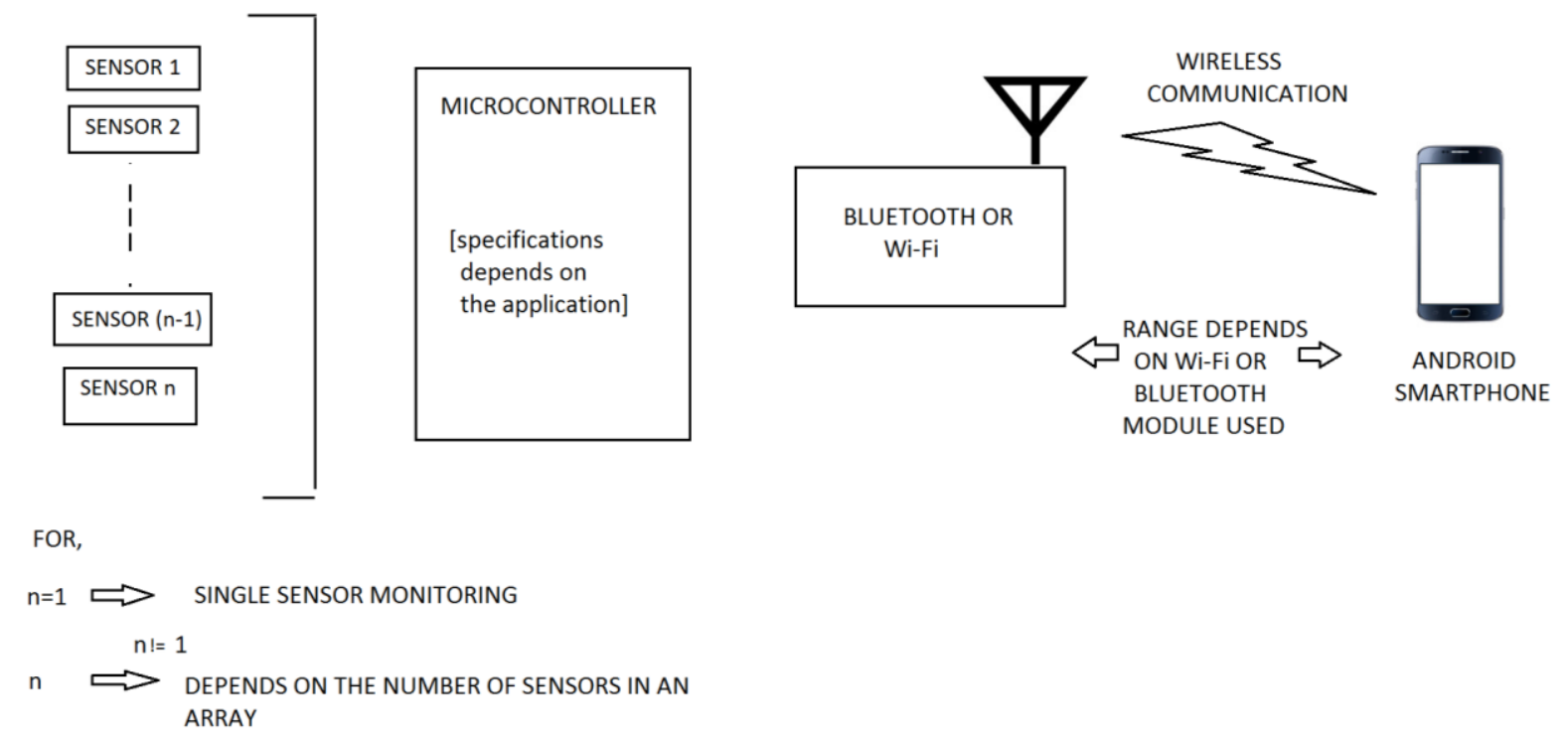

Figure 2 Block diagram of wireless monitoring of sensor values using Smartphone

\section{STEPS INVOLVED IN MOBILE APPLICATION DEVELOPMENT}

1. Requirement analysis: This is the first step in SDLC model to be followed. A thorough requirement analysis is carried out before design and development of the application.

2. Feasibility study and Historical analysis: Identification of certain fields where this application can be utilized or made use of. Also, a comparison with existing software/applications is done.

3. Design and implementation:

a) Flowchart/Algorithm

b) Code/blocks

c) Implementation

d) Integration and testing (Debugging)

e) Operating and maintenance of the application

f) Market and commercialize 


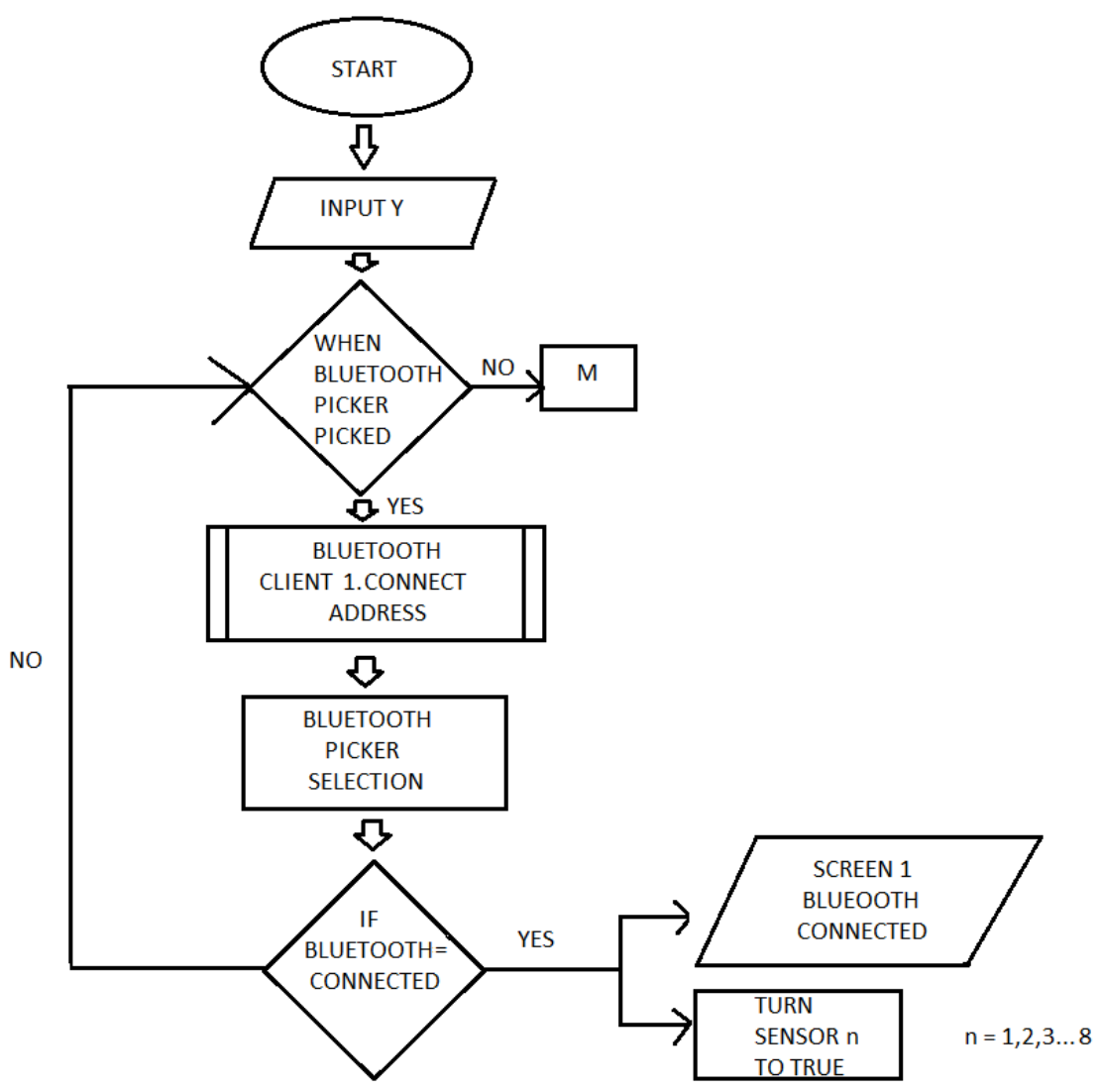

Figure 3 Flowchart when Bluetooth picker is picked

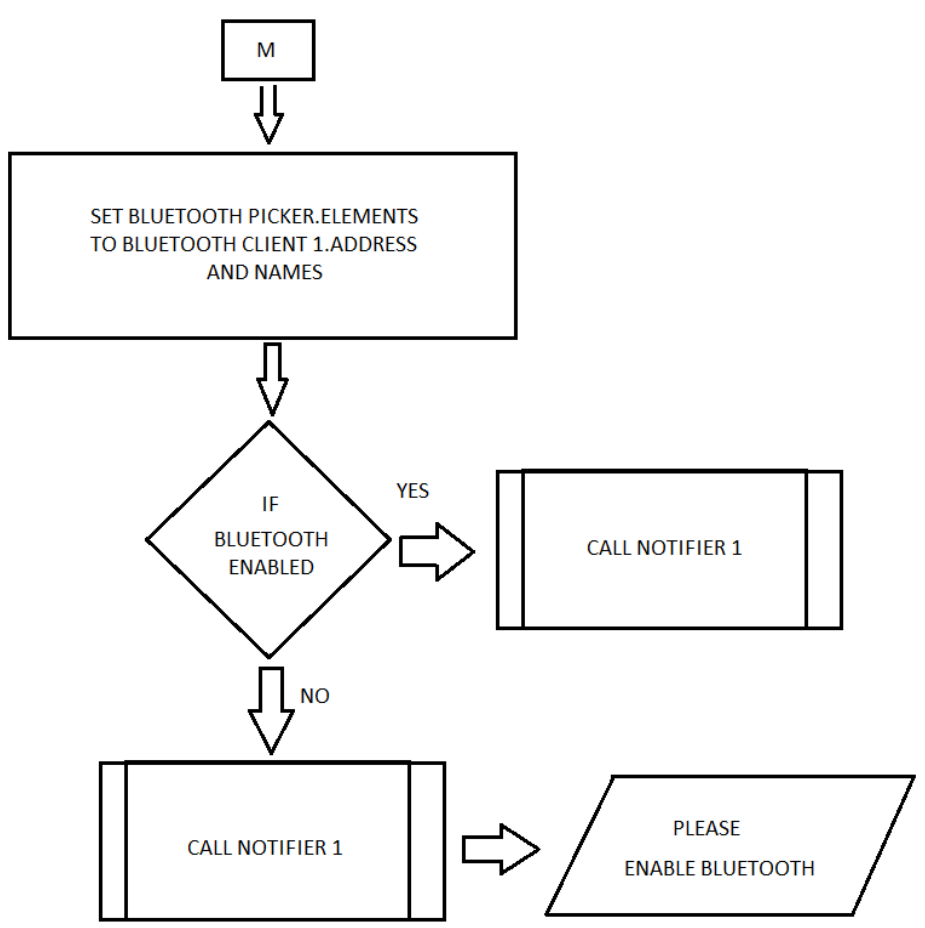

Figure 4 Flowchart before Bluetooth picker is picked 


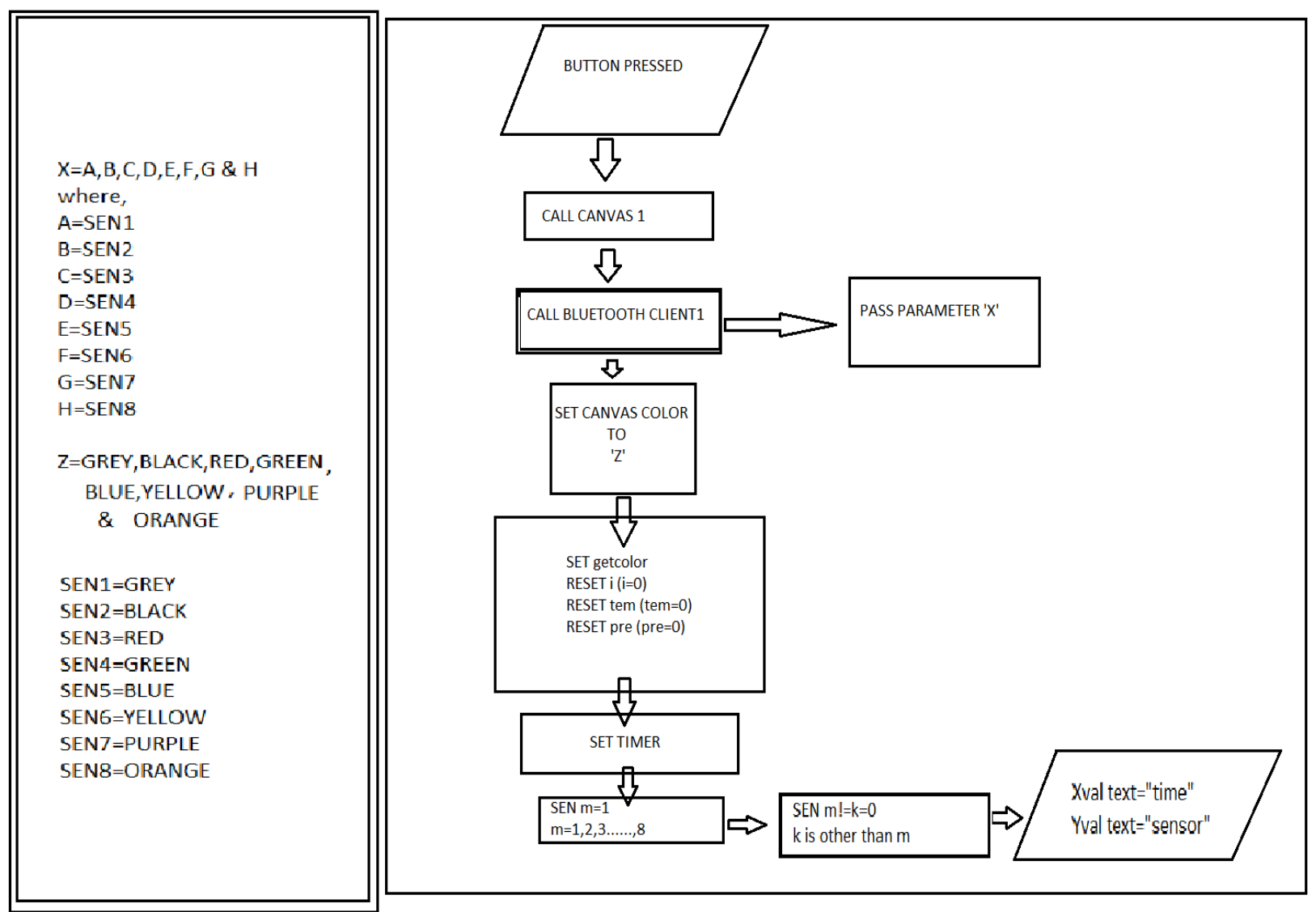

Figure 5 Flowchart when a button is pressed

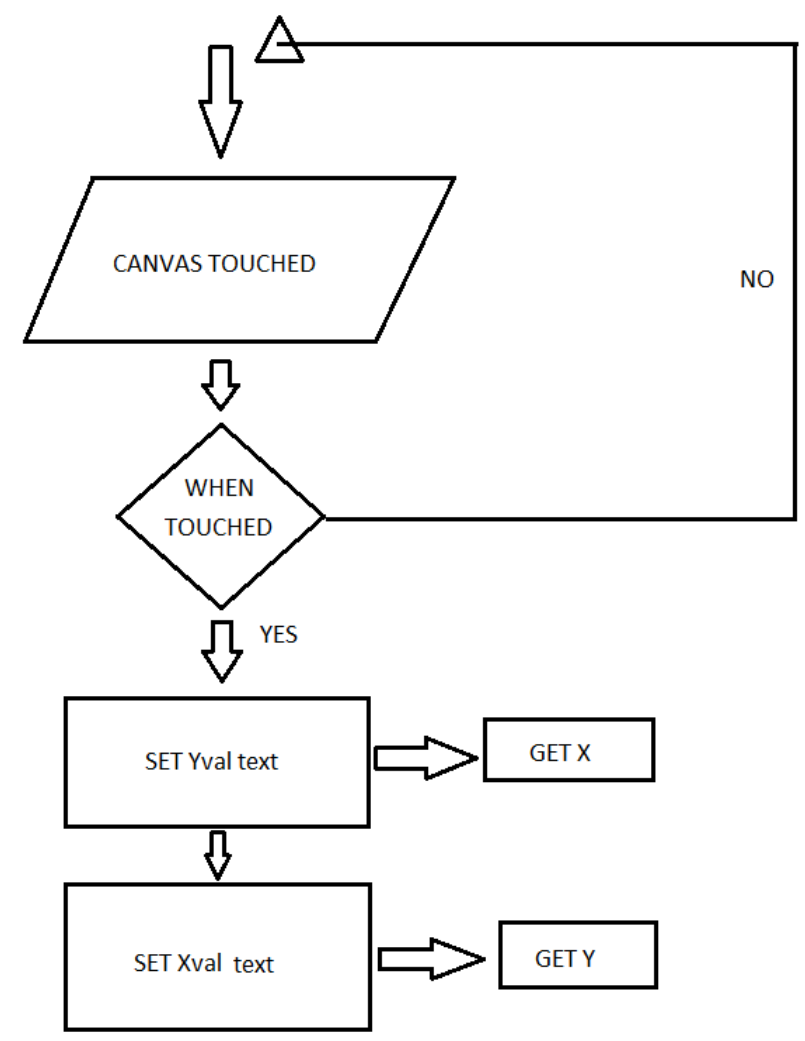

Figure 6 Canvas Procedure 
International Journal of Advanced Research in Computer and Communication Engineering ISO 3297:2007 Certified

Vol. 6, Issue 5, May 2017

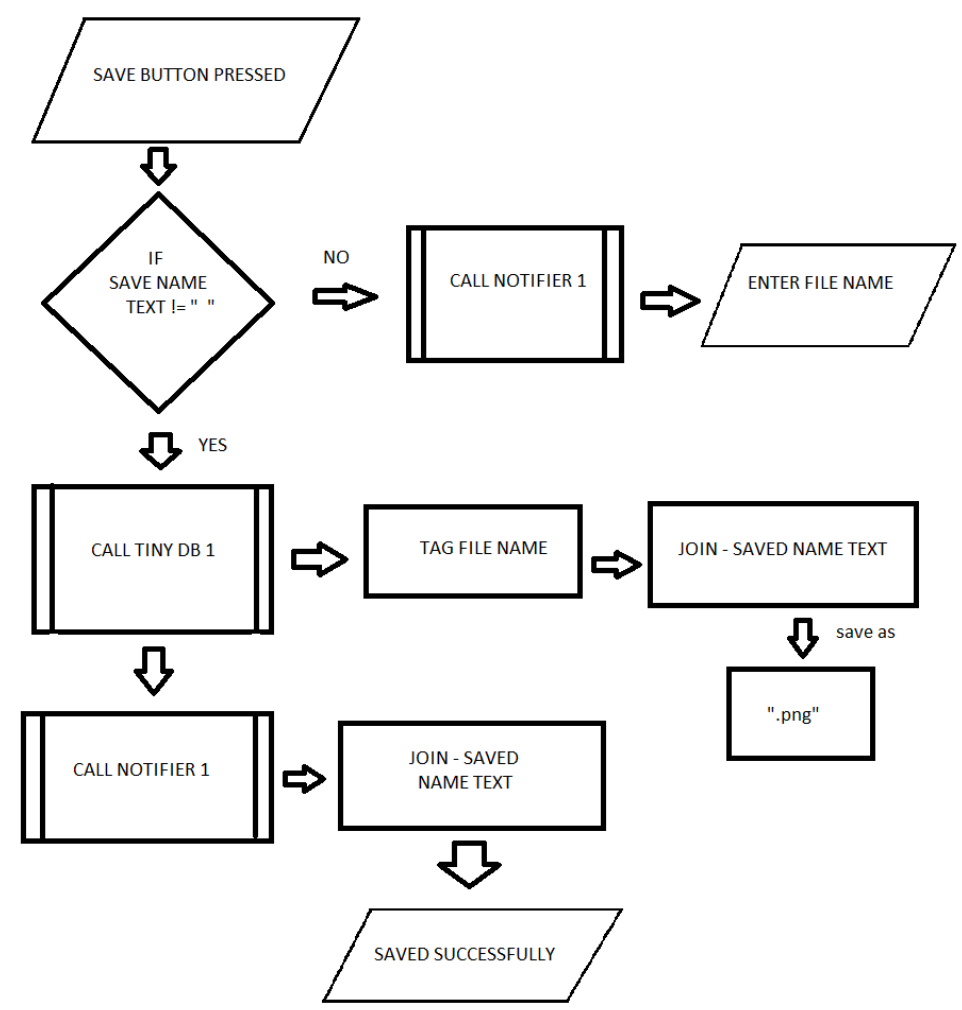

Figure 7 Events that take place when save button is pressed

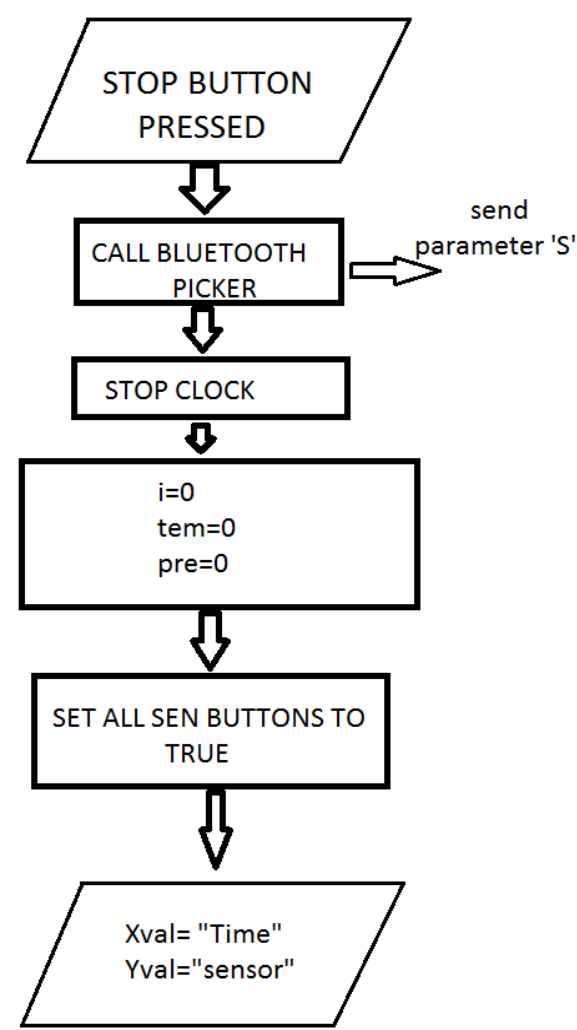

Figure 8 Events that occur when stop button is pressed 


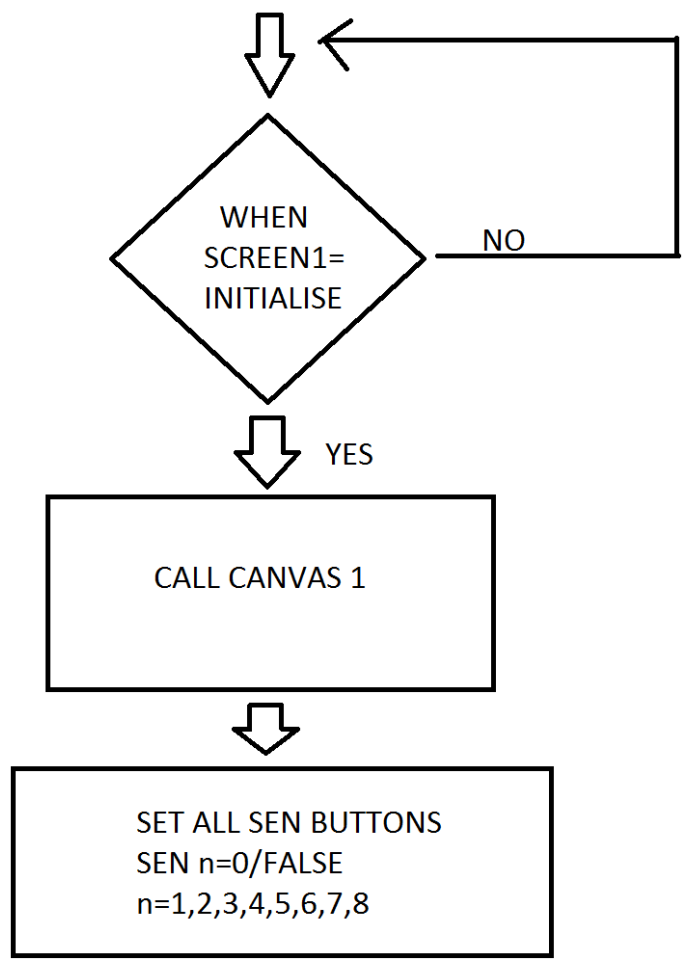

Figure 9 flowchart of initialization procedure

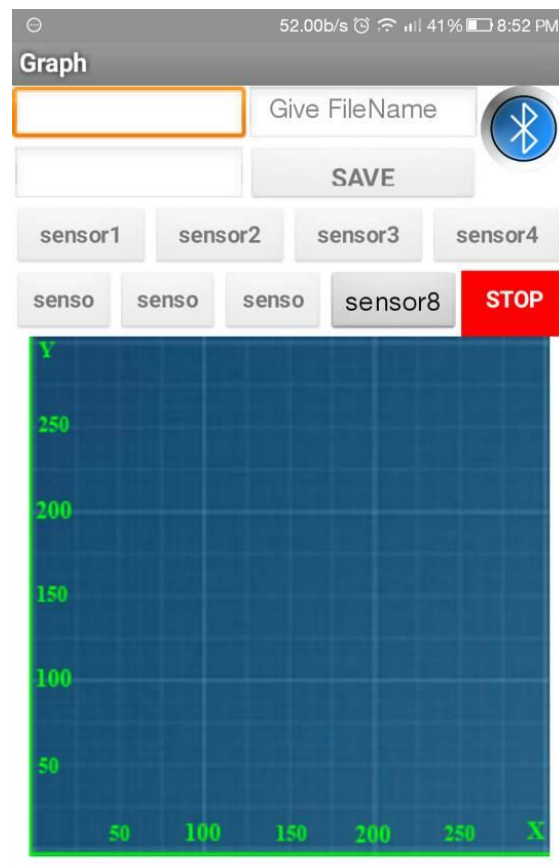

Figure 10 Screenshot of the application

\section{III.REQUIREMENT ANALYSIS}

Requirement analysis is the first stage of the SDLC model/software development process. Requirement analysis in software engineering encompasses those tasks that determine the needs of a new or altered product, satisfying the needs of beneficiaries or users.

There are 3 major steps followed in requirement analysis

- Eliciting requirements: This is also called requirement gathering. It involves communicating with customers, beneficiaries or users to determine their requirements 
- Analyzing requirements: Determining whether the stated requirements are unclear, incomplete, ambiguous or contradictory and resolving these issues

- Recording requirements

In this project, we require

- Sensor inputs

- Suitable microcontroller [6]

- Bluetooth [7] or Wi-Fi model [8]

- Smartphone (Running on Android OS)

- Good storage capacity and internal or external memory card

\section{IV.FEASIBILITY STUDY AND HISTORICAL ANALYSIS}

The feasibility study is used to justify a project. The main aim of a feasibility study is to discover and uncover the strengths and weaknesses of the software/application to be developed. Also, a historical analysis is carried out to uncover the strengths and weaknesses of similar software products of the past that need to be updated or replaced.

Steps followed in a feasibility study are:

- Determine implementation alternatives

- Asses the economic feasibility of each alternative

- Asses the technical feasibility of each alternative

- Asses the Operational feasibility of each alternative

- Choose and alternative

\section{DESIGN AND IMPLEMENTATION}

Before coding a flowchart/algorithm is constructed to solve/analyse the problem in a systematic manner. This also makes testing and debugging easier. Figure 3, shows the flowchart- when Bluetooth picker is picked.Figure 4 shows the flowchart- Bluetooth picker before picking.Figure 5 shows the flowchart when a button is pressed.Figure 6 shows canvas procedure.Figure 7 shows the events that take place when save button is pressed. Figure 8 shows events that occur when STOP button is pressed. Figure 9 shows the flowchartof initialization procedure.

\section{VI.RESULTS AND FINDINGS}

The android application for wireless monitoring of real time sensor values was successfully built and tested. The flowcharts in the previous sections provide a glimpse of the android application we have developed. A detailed report of the readings and observations of the application in real time will be presented in the next paper. Figure 10 shows the screenshot of the application being developed in KonigtronicsPvt. Ltd.

\section{CONCLUSIONS}

We are living in a period where the Smartphones have almost replaced the traditional personal computers when it comes to mobility, processing power, provision to develop and operate third party application. A single hand held device has the capability to perform similar to yesterday's super computers- even the most basic model has the access to more number- crunching capability than NASA had when it put men on the moon in 1969 [2] and it applies to ordinary human interactions.

\section{REFERENCES}

[1] Mobile phones: The rise of the cheap smartphone | The Economist- www.economist.com/.../21600134-smartphones-reach-masses-hostvendors-are-eager

[2] Smartphones: Planet of the phones | The Economist-http://www.economist.com/news/leaders/21645180 smartphone ubiquitous addictive and transformative planet phones

[3] Smartphones are becoming faster and cheaper | PCWorld-www.pcworld.com/article/.../lte-smartphones-are-becoming-faster-and-cheaper.html

[4] The Linux Kernel Archives-https://www.kernel.org/

[5] Welcome to iOS - Official Apple Support-https://support.apple.com/ios

[6] Arduino-https://www.arduino.cc/

[7] HC Serial Bluetooth Products User Instructional Manual - Makezinecdn.makezine.com/uploads/2014/03/hc_hc-05-user-instructionsbluetooth.pdf

[8] ESP8266EX Datasheet - Espressif Systems- espressif.com/sites/default/files/documentation/0a-esp8266ex_datasheet_en.pdf 


\section{BIOGRAPHIES}

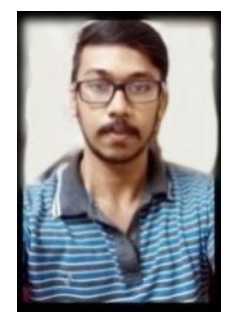

VISHESH S born on $13^{\text {th }}$ June 1992, hails from Bangalore (Karnataka) and has completed B.E in Telecommunication Engineering from VTU, Belgaum, Karnataka in 2015. He also worked as an intern under Dr Shivananju BN, Department of Instrumentation, IISc, Bangalore. His research interests include Embedded Systems, Wireless Communication and Medical Electronics. He is also the Founder and Managing Director of the company Konigtronics Private Limited. He has guided over a hundred students/lecturers/interns/professionals in their research works and projects. He is also the co-author of many International Research Papers. Presently Konigtronics Private Limited has extended its services in the field of Real Estate, Webpage Designing and Entrepreneurship.

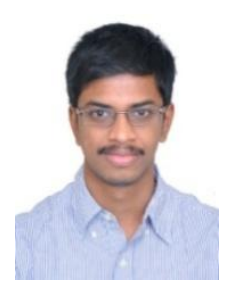

MANU SRINATH hails from Bangalore (Karnataka); he has completed B.E in Telecommunication Engineering from VTU, Belgaum, Karnataka. His research interests include networking, image processing and cryptography. He is the Executive Officer at the company Konigtronics (OPC) Pvt. Ltd.

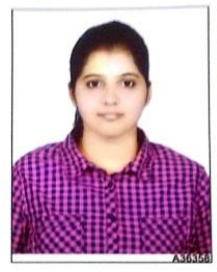

RANJITHA RAVISHANKAR hails from Bengaluru, Karnataka.She is currently pursuing B.E in Telecommunication and Engineering at B.N.M. Institute of Technology. Her areas of interests include Wireless communication, Computer Networks, Android development and Medical Electronics.

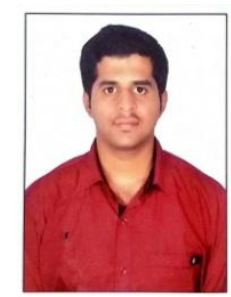

RANJAN RAVISHANKAR born on 19th September, 1995 hails from Bengaluru, Karnataka. He is currently pursuing B.E in Computer Science Engineering at Atria Institute of Technology, Bengaluru. His area of interest includes Computer Networks, Android Development, and Internet of Things.

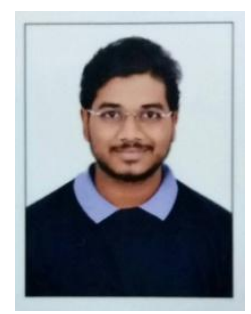

ASHRITH PRADEEP hails from Bengaluru, Karnataka. He is currently pursuing B.E in Computer Science and Engineering, under Visvesvaraya Technological University. His research interests include Embedded Systems and Internet of Things.

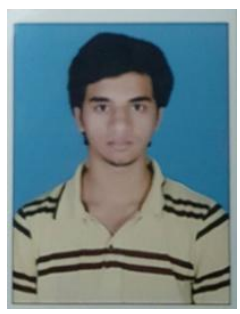

JEEVAN HS hails from Bengaluru, Karnataka. He is currently pursuing B.E in Information Science and Engineering at BNM Institute of Technology, Bengaluru. His research interests include Data Mining and Web Development. 